\title{
Identity tags: A vector for cross-infection?
}

\author{
S G Cox, ${ }^{1}$ MB ChB, FCS (SA), Cert Paed Surg (SA); A Burahee; ${ }^{2}$ A Lucier; ${ }^{3}$ C Fernando; $;$ \\ S M Machoki, ${ }^{1}$ MB ChB, MMed (Surg), FC Paed Surg (SA) \\ ${ }^{1}$ Department of Paediatric Surgery, Red Cross War Memorial Children's Hospital and University of Cape Town, South Africa \\ ${ }^{2}$ Medical student, University of Birmingham Medical School, UK \\ ${ }^{3}$ Medical student, University of North Carolina, Chapel Hill, North Carolina, USA
}

Corresponding author: S G Cox (sharon.cox@uct.ac.za)

\begin{abstract}
Background. Nosocomial infections represent one of the challenging problems of modern medicine. Healthcare providers play an important role in the transmission of these infections on their hands, clothing and equipment. Modern security systems require personnel to wear clearly displayed identity (ID) tags, and to have an easily accessible access disc. These access and ID tags are often worn around the neck on a lanyard, and could possibly harbour bacteria and be a vector for cross-infection.

Method. Saline-moistened swabs of the front and back of ID tags of 50 healthcare workers were taken for bacterial culture. Swabs were inoculated onto standard microbiological media. Potential pathogens were subjected to sensitivity testing while organisms resembling normal skin commensals were reported as such.

Results. Twenty-eight of the 50 (56\%) ID swabs cultured exhibited no bacterial growth. Eighteen (36\%) swabs grew primarily skin flora. Neutrophils were observed under microscopy on two (4\%) swabs. Seven (14\%) swabs grew potentially pathogenic bacteria. Doctors were found to have almost three times the risk of carrying pathogenic bacteria on their ID tags compared with nurses. Recent patient contact also showed a higher incidence of colonisation. There were no statistically significant differences between variables such as ward or area of work, nature of patient contact, time since qualification, level of qualification or length of employment at Red Cross War Memorial Children's Hospital, Cape Town, South Africa.

Conclusions. Prevention of hospital-acquired infections is important in any setting. The ID tag has been identified as a possible source of infection spread in this and previous studies. The ID tag has to date been neglected as a potential source of pathogen spread, and efforts to make staff aware of this potential danger should be considered in every institution.
\end{abstract}

S Afr Med J 2016;106(5):494-496. DOI:10.7196/SAMJ.2016.v106i4.9949

Nosocomial infections, especially those involving resistant microorganisms, represent one of the challenging problems of modern medicine. Healthcare providers play an important role in the transmission of these infections. White coats, neckties and stethoscopes are among the culprits implicated as vectors for transmission of infections by healthcare providers. ${ }^{[1-3]}$ Both pathogenic and nonpathogenic bacteria commonly colonise these items and simple infection-control measures such as strict hand hygiene, specific dress codes for staff and the bare-below-the-elbow policy often help in the fight against nosocomial infections. ${ }^{[4-6]}$

A number of publications have reported on the bacterial colonisation of white coats, neckties and stethoscopes and their possible role in crossinfection. ${ }^{[7]}$ With modern security systems in place in most hospitals, it has become important to wear clearly displayed identity (ID) tags, and to have an easily accessible access disc. At Red Cross War Memorial Children's Hospital (RCWMCH), Cape Town, South Africa (SA), as at many other institutions worldwide, these access and ID tags are worn around the neck on a lanyard.

As items of clothing have been shown to be a possible source of transmission of infections within hospital settings, it is reasonable to speculate that ID tags, worn on lanyards around the necks of healthcare workers, could harbour a similar bacterial profile and thus may be a vector for cross-infection. In addition, these ID tags are colourful, interesting objects, often used for distraction of an unhappy toddler while a procedure or examination is being performed; this makes the risk even greater in a paediatric setting.

\section{Objectives}

To determine the extent of colonisation of ID tags worn around the necks of healthcare workers at RCWMCH, and to describe the organisms isolated from these ID tags.

\section{Methods}

Permission was obtained from local and university ethics review boards and hospital management to perform the study prior to commencement (University of Cape Town Human Research Ethics Committee Ref. 492/2012). Participation of staff members was voluntary and anonymous. Informed consent was obtained from all participants. Swabs for bacterial culture were taken from the ID tags of 50 healthcare workers at RCWMCH, the largest tertiary hospital dedicated entirely to children's healthcare in SA. To achieve a cross-sectional representation of the different specialities, staff members in different units were approached. These areas included the intensive care unit (ICU), surgical wards, general medical wards and gastroenteritis ward. All staff present on the ward at the time of sampling, wearing an ID tag on a lanyard, were asked to participate (maximum of 50 participants).

Swabs were moistened with sterile saline, and the surface (front and back) of the ID tag, as well as the corners of the tag holder, was swabbed in a criss-cross manner.

Swabs were inoculated onto standard microbiological media at the National Health Laboratory Services (NHLS microbiology) located at Groote Schuur Hospital. Only potential pathogens (Staphylococcus aureus and Gram-negative bacilli) were followed up; organisms resembling normal skin commensals were reported as such.

Data were summarised as total numbers (column percentages) for categorical variables. Baseline comparisons where appropriate were made using a $\chi^{2}$ test. Statistical significance was determined as $p<0.05$. All statistical analyses were performed using SPSS Statistics, version 21 (IBM, USA).

\section{Results}

Control group

Cultures from 10 unused ID tags exhibited no bacterial growth. 


\section{Participant demographics}

The ID tags of 21 doctors and 29 nurses were swabbed. Table 1 reflects the results relating to participant demographics, as well as patient contact, ID-tag cleaning practices and time worked at the institution.

\section{Bacteria}

Twenty-eight of the 50 (56\%) ID-tag swabs cultured exhibited no bacterial growth. Eighteen (36\%) swabs grew primarily skin flora. Neutrophils were observed under microscopy on two (4\%) swabs.

Seven (14\%) swabs grew potentially pathogenic bacteria comprising coagulase-negative Staphylococcus $(n=1)$, Enterobacter cloacae with an inducible beta-lactamase and Klebsiella $(n=1)$, Micrococcus spp. $(n=1)$, Gram-positive cocci $(n=2)$, Gram-positive bacilli $(n=2)$ and Gram-negative bacilli $(n=2)$. One culture grew a few mixed aerobic organisms as well as Gram-negative bacilli. None of the cultures exhibited anaerobic organism growth. While doctors and nurses had elicited different proportions of contaminations, a comparison using a $z$-score revealed no significant difference $(z$-score $-1.70 ; p=0.09)$.

\section{Decontamination practices}

Decontamination practices were observed among 26 of the participants $(52 \%)$. A total of 24 of the staff members had never cleaned their ID tag, 18 cleaned it $<1$ day a week, and 8 cleaned it more often.

Of the participants whose swabs cultured pathogenic bacteria, four had never cleaned their ID tag, all had had contact with a patient in the previous hour, and notably, two of the four worked on the gastroenteritis ward.

There was no significant correlation identified between participants with pathogenic microbial growth on culture and measured variables such as ID-tag cleaning frequency. However, there was a positive correlation of positive pathogenic growth with participants sampled within 30 minutes of patient contact ( $p=0.006$ ). No further correlations were identified between these participants and other variables previously mentioned.

\section{Risk of carrying pathogens}

Doctors were found to have almost three times the risk of carrying pathogenic bacteria on their ID tags compared with nurses, although this was not significant (relative risk (RR) 2.98; 95\% confidence interval (CI), 0.63 - 14.1; $p=0.17$ ).

There were no statistically significant differences between other variables, such as ward or area of work, nature of patient contact, time since qualification, level of qualification or length of employment at RCWMCH (estimated time of ID-tag use).

\section{Discussion}

The paediatric patient population at $\mathrm{RCWMCH}$ makes it very probable that ID tags hanging around the necks of physicians to the waist level inadvertently come into contact with patients and the clinical environment. On occasion, they are used as a distraction to facilitate consultation. It may be reasonably assumed that these processes and the regular need to apply the tag to the security panel may result in the tags ultimately becoming colonised with potential nosocomial pathogens.

Previous studies have documented that many articles of clothing worn by healthcare staff can harbour potential pathogens such as methicillin-resistant $S$. aureus (MRSA) and $S$. aureus on doctors' neckties ${ }^{[8,9]}$ and MRSA on stethoscopes. ${ }^{[10]}$ Contaminated equipment and clothing provide a reservoir from which healthcare workers may reinoculate their hands after hand hygiene practices have been
Table 1. Participant demographics and results

\begin{tabular}{lll}
\hline & Doctors, $\boldsymbol{n}$ (\%) & Nurses, $\boldsymbol{n}$ (\%) \\
\hline Unit & & \\
$\quad$ Surgical speciality ward & $1(4.8)$ & $4(13.8)$ \\
General surgery and urology & $9(42.9)$ & $3(10.3)$ \\
General medical ward 1 & $2(9.5)$ & $3(10.3)$ \\
General medical ward 2 & 0 & $3(10.3)$ \\
ICU & $4(19.0)$ & $7(24.1)$ \\
Gastroenteritis ward & $5(23.8)$ & $9(31.0)$ \\
Tag-cleaning frequency & & \\
Never & $13(61.9)$ & $11(37.9)$ \\
$<25 \%$ of time & $6(28.6)$ & $12(41.4)$ \\
$25-75 \%$ of time & $2(9.5)$ & $3(10.3)$ \\
Always & 0 & $3(10.3)$ \\
Nature of last patient contact & & \\
Intact skin only & $15(71.4)$ & $18(62.1)$ \\
Invasive procedure & $3(14.3)$ & $3(10.3)$ \\
Mucous membranes, secretions & $3(14.3)$ & $2(6.9)$ \\
Nappy change, stool contact & 0 & $6(20.7)$ \\
Time since last patient contact \\
(minutes)
\end{tabular}

carried out. The British Medical Association has suggested that doctors refrain from wearing non-essential items of clothing such as ties and implement a bare-below-the-elbow policy. ${ }^{[1]}$

The relatively low prevalence of both methicillin-susceptible $S$. aureus (MSSA) and MRSA in this study compared with other international studies ${ }^{[1,5]}$ can be explained by a previous article from this institution, ${ }^{[12]}$ documenting both the low annual incidence of bacteraemia at 3.28 cases per 1000 hospital admissions and the fact that MRSA was responsible for only $26 \%$ of S. aureus bacteraemia. In this article, only six possible cases of community-acquired MRSA infections were described. ${ }^{[12]}$

It has previously been shown that lanyards were particularly contaminated, with a median bacterial load per unit surface area up to ten times greater than ID tags. ${ }^{[13]}$ Studies have also shown that bacteria are able to survive on plastic surfaces for long periods of time, with Gram-negative bacteria surviving for $>60$ days and 
enteroccoci for $>90$ days. ${ }^{[14]}$ Hence, our study focused on ID tags as a separate entity.

We showed that decontamination practices by $52 \%$ of staff were more prevalent than in previous reports (16\% and $27 \%) .{ }^{[15,16]}$ The tags that had never been cleaned were more likely to carry pathogenic growth. Also, there was a significant positive likelihood of pathogenic growth within the first 30 minutes of patient contact, irrespective of the nature of the patient contact. Hence, we recommend that an effective decontamination regimen (e.g. 30 seconds criss-cross scrubbing with a cleaning swab) be implemented where ID tags may have been in contact with patients.

While there was a higher risk that doctors' tags were colonised with potential pathogens, there was not a significant difference between doctors and nurses, contrary to what has previously been reported. ${ }^{\left[{ }^{13]}\right.}$ The equal compliance with hand-hygiene protocols observed by both nurses and doctors at RCWMCH may help to explain the lack of difference in contamination rates observed in our study. However, larger studies are required to compare the relative carriage rate of potential pathogens between doctors and nurses.

\section{Study limitations}

One limitation of our study is that we did not assess the participants for carriage of $S$. aureus in the nares or on their hands. Also, we did not do a baseline evaluation of the current pathogenic bacterial occurrence on the ward at the time of data collection. Hence, we were unable to correlate between inoculated bacteria from the swabs and the clinical occurrence on the wards. Another limitation is that although staff members estimated the length of time for which they had been using the ID tags, we were unable to establish the exact point of contamination.

\section{Conclusion}

Prevention of hospital-acquired infections is important in any setting. Efforts to reduce these infections include the use of protective clothing, a no-sleeve policy, and, most importantly, hand washing. The ID tag has been identified as a possible source of infection spread in previous studies. This study shows that most staff either seldom or never clean their ID tags, and this results in a higher incidence of tag colonisation. It shows a higher probability of the tag being infected if the tag owner is a doctor (although not significant), or has had recent patient contact. Most hospitals have education drives, strict handwashing practices and sanitising equipment available. The ID tag has to date been neglected as a potential source of pathogen spread and efforts to make staff aware of this potential danger should be considered in every institution.

Acknowledgement. The study was funded by the University of Cape Town School of Child and Adolescent Health Research Award.

\section{References}

1. Koh K, Husni S, Tan JE, et al. High prevalence of methicillin-resistant Staphylococcus aureus (MRSA) on doctors' neckties. Med J Malaysia 2009;64(3):233-235.

2. Srinivasan M, Uma A, Vinodhkumaradithyaa A, Gomathi S, Thirumalaikolundusubramanian P. The medical overcoat - is it a transmitting agent for bacterial pathogens? Jpn J Infect Dis 2007;60(2-3):121-122.

3. Youngster I, Berkovitch M, Heyman E, Lazarovitch Z, Goldman M. The stethoscope as a vector of infectious diseases in the paediatric division. Acta Paediatr 2008;97(9):1253-1255. DOI:10.1111/ j.1651-2227.2008.00906.x

4. Bandi, S, Uddin L, Milward K, Aliyu S, Makwana N. How clean are our stethoscopes and do we need to clean them? J Infect 2008;57(4):355-356. DOI:10.1016/j.jinf.2008.07.018

Hill C, King T, Day R. A strategy to reduce MRSA colonization of stethoscopes. J Hosp Infect Hill C, King T, Day
2006;62(1):122-123.

6. McGovern B, Doyle E, Fenelon LE, FitzGerald S. The necktie as a potential vector of infection: Are doctors happy to do without? J Hosp Infect 2010;75(2):138-139. DOI:10.1016/j.jhin.2009.12.008

7. Weber R, Khan P, Fader R, Weber R. Prospective study on the effect of shirt sleeves and ties on the transmission of bacteria to patients. J Hosp Infect 2012;80(3):252-254. DOI:10.1016/j.jhin.2011.12.012

8. Ditchburn I. Should doctors wear ties? J Hosp Infect 2006;63(2):227-228. DOI:10.1016/j. jhin.2006.01.027

9. Lintott P, Parry D. Let's lose the tie. J Hosp Infect 2001;48(1):81-82. DOI:10.1053/jhin.2000.0902

0. Madar R, Novakova E, Baska T. The role of non-critical health-care tools in the transmission of nosocomial infections. Bratisl Lek Listy 2005;106(11):348-350.

1. British Medical Association (BMA). Health Care Associated Infections: A Guide to Healthcare Professionals. London: BMA, 2006:13.

12. Naidoo R, Nuttall J, Whitelaw A, Eley B. Epidemiology of Staphylococcus aureus bacteraemia at a tertiary children's hospital in Cape Town, South Africa. PLoS One 2013;8:e78396. DOI:10.1371/ journal.pone.0078396

13. Kotsanas D, Scott C, Gillespie E, Korman T, Stuart R. What's hanging around your neck? Pathogenic Kotsanas D, Scott C, Gillespie E, Korman T, Stuart R. What's hanging
bacteria on identity badges and lanyards. Med J Aust 2008;188(1):5-8.

14. Neely A. A survey of Gram-negative bacteria survival on hospital fabrics and plastics. J Burn Care Rehabil 2000;21(6):523-527. DOI:10.1097/00004630-200021060-00009

15. Pepper T, Hicks G, Glass S, Philpott-Howard J. Bacterial contamination of fabric and metalbased identity card lanyards: A cross-sectional study. J Infect Public Health 2014;7(6):542-546. DOI:10.1016/j.jiph.2014.07.009

16. Alexander R, Volpe N, Catchpole C, Allen R, Cope S. Are lanyards a risk for nosocomial transmission of potentially pathogenic bacteria? J Hosp Infect 2008;70(1):92-93. DOI:10.1016/j.jhin.2008.05.022

Accepted 18 March 2016. 